\title{
EL HALLAZGO DE UN QUIPUCAMAYOC EN CERRO COLORADO, HUACHO
}

\author{
Pieter D. VAN DALEN LUNA \\ UniversidAd NACIONAL MAYOR DE SAN MARCoS \\ pvandalen2@hotmail.com
}

\section{RESUMEN}

Se presentan los resultados de los análisis de un quipu recuperado del interior del fardo funerario de un infante. Este personaje habría sido un quipucamayoc, quien en su crecimiento aprendería el oficio de administrador contable en este quipu, que no presenta nudo alguno.

Palabras claves: arqueología, cultura Chancay, quipu, Tawantinsuyu, Huacho, costa central.

\section{Abstract}

We present the results of the analysis of a quipu recovered from the interior of an infant's funeral bundle. This personage would have been a quipucamayoc, who in its growth would learn the office of accounting administrator in this quipu, that does not present any knot.

Keywords: archeology, Chancay culture, quipu, Tawantinsuyu, Huacho, central coast.

\section{INTRODUCCIÓN}

Hasta el momento son escasos los quipus recuperados de contextos arqueológicos en la Costa Norcentral peruana. Es importante por ello, el hallazgo de un neonato con un quipu al interior de su fardo, procedente del área funeraria de Cerro Colorado, en el valle de Huaura. Este territorio fue ocupado por la cultura Chancay en periodos prehispánicos tardíos, siendo anexado al Tawantinsuyu durante el cogobierno de Pachacuteq y Tupac Yupanqui.

No hay evidencias sobre presencia de grupos mitqmas en las zonas arqueológicas aledañas ni entre los entierros de Cerro Colorado, por lo que planteamos que la población enterrada en esta área funeraria y la que vivió en los alrededores fueron de la cultura Chancay. Sin embargo, existió en el valle, personal administrativo designado por el estado cusqueño para actividades de control de la población local, entre estos los quipucamayoc. 


\section{El Complejo Funerario de Cerro Colorado}

El complejo funerario de Cerro Colorado es el área funeraria más extensa de la Costa Central Peruana, ubicado en el extremo meridional del valle bajo del río Huaura, en el cono de deyección de una quebrada de medianas dimensiones que se une al valle, sobre un terreno arenoso depositado sobre formaciones pétreas de coloración rojiza. Políticamente se ubica en la localidad de Los Pinos (ex Asentamiento Humano Alberto Fujimori), distrito de Santa María, provincia de Huaura, región Lima.

La extensión de este cementerio abarcaba entre el Cerro Colorado y gran parte del sector meridional del valle de Huaura, extendiéndose hasta la Playa Colorado, territorio hoy ocupado por numerosos Asentamientos Humanos y el área de la ciudad universitaria de la Universidad Nacional José Faustino Sánchez Carrión. Así pues, esta era el área de enterramiento de los antiguos pobladores del valle de Huaura, pues mientras los grandes centros políticos administrativos y residenciales se localizaban en medio del valle, como el complejo arqueológico Walmay, su población era enterrada en esta gran área funeraria en medio de grandes rituales.

A pesar que casi no hay arqueólogo que no haya escuchado hablar o haya visitado este complejo de Cerro Colorado, nadie ha realizado investigación arqueológica alguna, pues solo se conocen sus materiales a partir de proyectos de evaluación y rescates arqueológicos (Tosso 1997, Rodríguez 2007, Morales 2009, van Dalen, Grados, Tello, Vivanco, Flores y Marcelo 2014, van Dalen y Carbonel 2015, van Dalen, Tello y Grados 2016) o por la acción del huaqueo.

El área más importante se encuentra ubicado hacia el lado este de la carretera Panamericana, inmediatamente al ingresar a la ciudad de Huacho, terreno que hoy se encuentra ocupado por numerosos asentamientos humanos, los cuales cada año van avanzando y ocupando parte del cementerio huaqueado.

Esta área está dividida en tres grandes sectores:

Sector del Tambo: Conformado por un conjunto de edificaciones de planta cuadrangular, construidas a base de tapiales, los cuales fueron finalmente pintados de color rojo y crema. Se trata del tambo por donde pasaron los primeros hispanos que se dirigían a Pachacamac a inicios de 1533.

Sector amurallado: Se trata de un conjunto de edificaciones ubicadas en la cima del Cerro Colorado que se encuentran circundadas por tres sistemas de murallas, edificadas a base de piedras canteadas y adobes.

Área Funeraria: Conformado por el extenso cementerio que contiene contextos funerarios con o sin estructuras funerarias.

El sitio en general se encuentra en mal estado de conservación debido al huaqueo y a las invasiones, encontrándose en estado de abandono por parte del Estado Peruano y el Ministerio de Cultura.

\section{El Proyecto Arqueológico Cerro Colorado}

En base al Proyecto de Evaluación Arqueológica desarrollada el año 2000 por Walter Tosso (2000) el complejo funerario fue dividido en cinco parcelas, de las cuales la primera quedaba como Zona Intangible; la segunda, tercera y cuarta quedaban como Zonas de Emergencia, por lo que debía realizarse trabajos de Rescate Arqueológico; y la quinta quedaba como Zona Desafectada. En tal sentido, entre los años 2014 y 2017 venimos realizando trabajos de excavación arqueológica en la Parcela 2 , en un área que abarca casi 15 cuadras de largo por 4 cuadras de ancho, en los cuales se ha podido recuperar cerca de dos millares de contextos funerarios de la cultura Chancay. Estas investigaciones 
en campo, desarrolladas mediante cuidadosos trabajos de excavación, fueron complementados en gabinete con el análisis de cada uno de los materiales recuperados, con participación de profesionales de diferentes especialidades y centros de investigación.

Las unidades de excavación (50) fueron realizadas en área y emplazadas a lo largo y ancho de todas las calles del área involucrada. Los trabajos de excavación se realizaron conjuntamente con los arqueólogos asistentes de campo y personal especializado de las mismas asociaciones de vivienda.

\section{El Hallazgo de un Quipucamayoc}

En el área de la Asociación de Vivienda Los Pinos I Etapa, se emplazó la Unidad de Excavación 25, la cual fue dividida en tres subunidades debido a su amplitud. Al interior de la capa A, se halló una capa de relleno de arena, de granulometría fina, de color marrón, presentando inclusión de piedras, basura moderna y restos culturales disturbados como cerámica, textiles, óseos, objetos hechos de restos malacológicos. Asimismo, se halló al interior de esta capa un conjunto de Hallazgos y Contextos Funerarios.

A una distancia de 18 metros al norte del extremo sur de la unidad y a dos metros al este del extremo occidental de la unidad se recuperó el hallazgo 29, a una profundidad de $1.48 \mathrm{~m}$ desde la superficie. Este hallazgo es el fardo funerario de un neonato, dispuesto en eje este-oeste en su mayor longitud. Consiste en un envoltorio textil atado con cordones de fibra de algodón, se observa parte del textil quemado en la base del envoltorio; además hacia los extremos se encuentran muy deteriorados por lo que se observa el algodón interno del fardo. Los colores predominantes del textil usado son marrones y cremas, el algodón es natural con semillas. Al retirar el fardo se observó en la base algunas vertebras no fusionadas de un neonato. Para colocar el fardo se elaboró una muy pequeña matriz simple, cavada en la arena.

El análisis en laboratorio del material recuperado evidenció que se trata de un fardo funerario pequeño de $0.42 \times 0.35 \mathrm{~m}$., con envoltorio textil de color marrón sin decoración en técnica simple de $1 \times 1$ trama-urdimbre y anudado con dos hilos trenzados a la altura del pecho y abdomen; el cráneo es muy laminar en proceso de osificación membranosa, mandíbula fracturada y periostio de color verde; el esqueleto está completo; los dos brazos y 2 piernas están disecadas, momificados con su piel de color cremoso, cubierto con abundante algodón fifo.

El individuo es de género masculino, bebé 0 a 3 meses de edad, con modelación cefálica tabular erecta, talla de $0.42 \mathrm{~m}$. El cráneo tiene metopismo. Los brazos y piernas han sido momificados con sal y algodón. Presenta Hiperostosis porótica generalizada en el cráneo (por anemia).

Al interior del fardo, el individuo tenía abundante algodón marrón alrededor del cráneo, brazos, tórax y abdomen. Hay una lámina de cobre incrustada en la boca. Tenía 4 choclos del maíz huachano ( 2 a cada lado del cuerpo, cerca de los brazos y envueltos en textiles y algodón fifo). Al interior del fardo, el individuo se hallaba en posición decúbito dorsal, con la cabeza destruida y los brazos y piernas flexionadas. Presenta un quipu sobre el pecho en proceso de elaboración.

\section{CARActerísticas del QuiPU}

El quipu está elaborado en fibra de algodón natural. Tiene un soporte grueso de $4 \mathrm{~mm}$ de grosor, formado por dos hilos con torsión en "Z" y retorsión en "S". El soporte tiene $62 \mathrm{~cm}$ de largo. De este soporte salen nueve conjuntos de hilos, los cuales presentan retorsión en "S" en el extremo superior, poco antes de insertarse al soporte. Cada conjunto de hilos está formado por entre seis y diez hilos, de color beige y elaborados en fibra de algodón, con un largo casi uniforme. No presenta nudos en ninguna parte, pues no fue utilizado. 


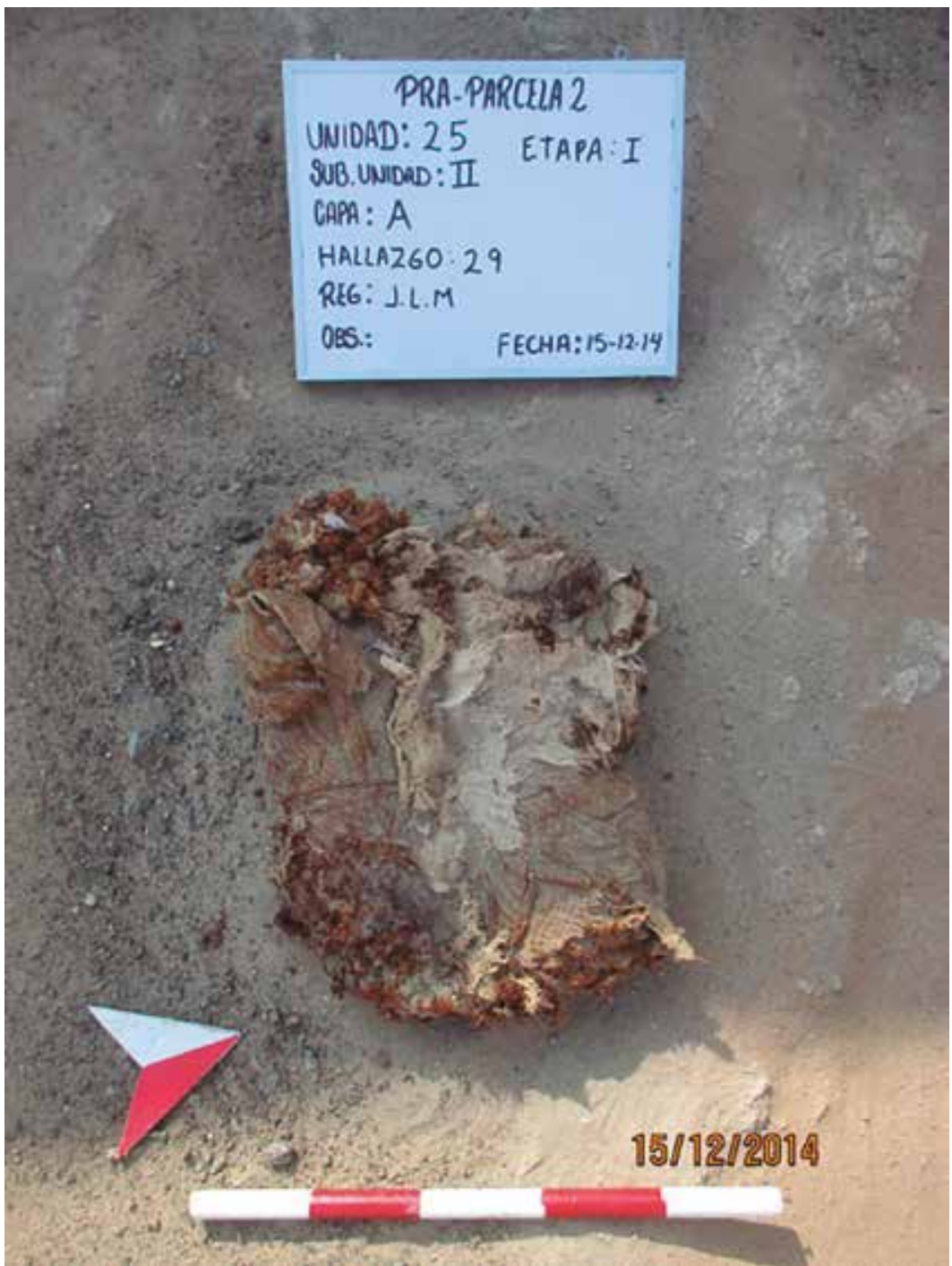

Figura 1: Hallazgo 29, fardo de bebe

\section{El Hallazgo de Quipus en la Costa Central y}

\section{el Quipucamayoc de Cerro Colorado}

Son escasos los casos en que se han recuperado quipus en contextos arqueológicos en la Costa Nor Central Peruana. Entre estos pocos casos debemos mencionar el caso del quipu de Caral, procedente de contextos precerámicos, lo cual sería la evidencia más antigua reportada en toda el área andina (La República, 16 de julio de 2005). En el valle de Huaura, se ha recuperado un quipu de un cementerio disturbado en Acaray, aunque hasta el momento no se ha reportado sus características. Sin embargo, en la década 40 del siglo pasado, Julio C. Tello a su paso por el valle de Huaura, describió dos quipus pertenecientes a la Colección Fumagali, los cuales posiblemente procedían de Cerro Colorado o Pam- 


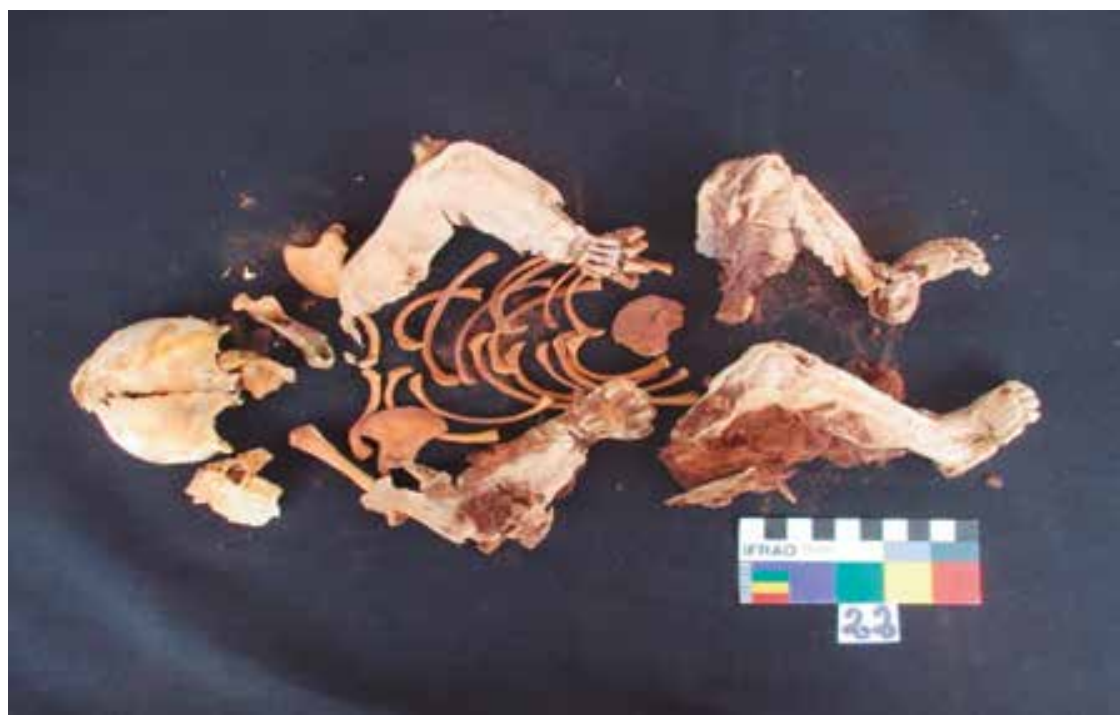

Figura 2: Momia del bebé de 1-3 meses de edad del Fardo n 15, nótese los tejidos momificados de los brazos y piernas de color cremoso. Consideramos que en el proceso de elaboración se colocaba sal y bastante algodón marrón.

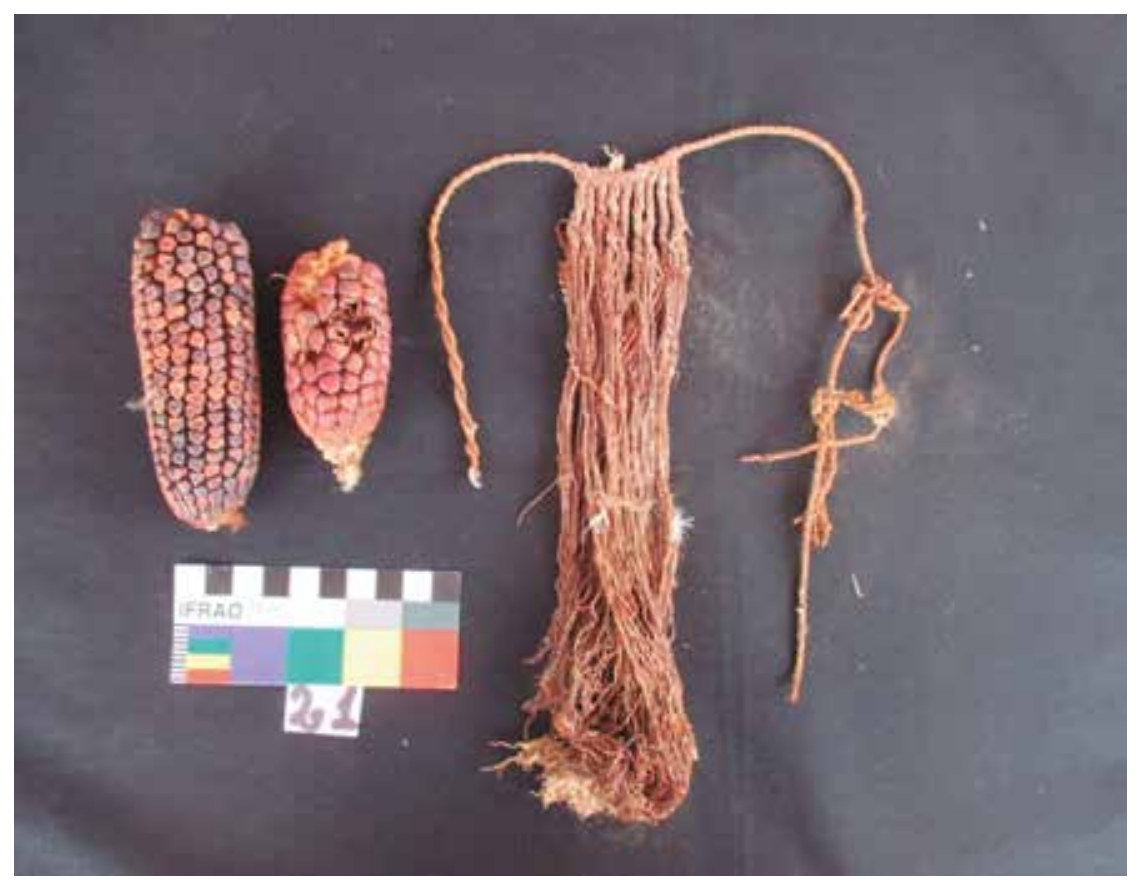

Figura 3: Contenido del Fardo a modo de ofrenda asociado al bebé con dos choclos huachanos y un quipu en proceso de elaboración. 
pa de Animas (Tello 2015: 50-51). El primer quipu de la colección Fumagalli descrito por Tello, consta de 53 hilos o cordones, con nudos simples y consecutivos, cuerdas de color añil y blanco; mientras que el segundo tiene 22 cuerdas de colores: amarillo, añil, blanco y rojo, también con nudos simples, consecutivos y en espiral. De igual manera Tello registra el hallazgo de otro pequeño quipu, pero esta vez procedente del valle de Chancay, posiblemente de algún sitio arqueológico de Huando o sus alrededores. Este quipu de cinco cuerdas se caracteriza por presentar un cordón de color negro. Algunos museos y coleccionistas privados cuentan en la actualidad con algunos ejemplares como Miguel Silva que tiene dos ejemplares procedentes de Cerro Colorado.

En el vecino valle de Chancay, el autor del presente artículo analizó un quipu procedente del sitio de Lauri, el cual también era de contexto desconocido (van Dalen 2007), caracterizado por presentar un soporte de madera tallada representando una mazorca de maíz de cuyo interior emergen hilos de diferentes colores y grosores. De igual manera en las excavaciones de la Temporada 2012-2013 en el complejo arqueológico de Lumbra se halló junto al Ushnu del Sector "B", en un estrato de relleno del Horizonte Tardío, dos fragmentos de un mismo quipu, con cuerdas hechas de algodón; mientras que, en una capa de relleno, en el interior de un recinto doméstico del sector A se hallaron dos cuerdas pertenecientes a un quipu, cada una con sus nudos (van Dalen y Grados 2015, van Dalen 2015: 16-17; 2016).

Los quipus son instrumentos mnemotécnicos, que servían para registrar datos en sus caracteres representados por nudos de diversas formas, los cuales tenían un valor característico. Los registros que se hacían eran diversos, como registrar historias, para información censal, registros legales y censos de producción agrícola, entre otros. Los nudos son elaborados con las mismas cuerdas colgantes o con subsidiarias insertadas. Los quipus están formados por numerosas cuerdas verticales, que afloran de una cuerda matriz de posición horizontal; sin embargo, cada cuerda puede tener numerosas cuerdas secundarias que se encuentran insertadas mediante nudos, las que a su vez tienen cuerdas terciarias y sucesivamente, según la complejidad que puedan tener. Existe toda una compleja tipologías de quipus, relacionados con su filiación temporal y cultural (Urton y Brezine 2009, Arellano 1999: 251).

El conocimiento y aplicación de los quipus fueron exclusivamente de competencia de los llamados "quipucamayoc", quienes eran los responsables del manejo de la contabilidad y las estadísticas en el imperio del Tawantinsuyu (van Dalen 2007: 226). Pero había dentro de los quipucamayoc, diferentes jerarquías. Rostworowski (1990:59) señala por ejemplo que el manejo de estos instrumentos estaba a cargo de un alto personaje que recibía las cuentas enviadas de distintas poblaciones situadas en una determinada área geográfica. Bajo las ordenes de estos quipucamayoc principales existían toda una serie de contadores menores y asistentes. Pero no solo el estado Inca contaba con estos sistemas contables, los curacas regionales llevaban también los registros de lo que contenían sus depósitos y de la gente que gobernaban.

El hecho de hallar a un neonato conteniendo al interior de su fardo un quipu sin evidencias de nudos, solo puede denotar el hecho que se trataba de un personaje que se iba a desempeñar como quipucamayoc cuando crezca, actividad muy posiblemente heredada de su padre y sus ancestros. El haber muerto a temprana edad hizo que se le entierre con su futuro instrumento de trabajo. Según Waldemar Espinoza (1997: 423) los quipucamayocs transmitían su conocimiento práctico y oral a sus herederos, siendo consideradas como personas altamente especializadas en el imperio del Tawaninsuyu. Carlos Radicati (2006: 136-137) tomando información de varios cronistas, también afirma que el cargo de los quipucamayocs era hereditario, siendo enseñado de padres a hijos, aunque también existía escuelas donde se enseñaba el oficio de hacer y leer quipus. 
Lo que resulta importante con el presente estudio, es comprobar que, si existió en el cementerio de Cerro Colorado, personajes de la sociedad Chancay dominados por el estado Tawantinsuyano, que fueron enterrados portando quipus; puesto que este no es el primer hallazgo, como ya vimos líneas arriba, hay otros autores que reportaron el hallazgo de quipus.

\section{Conclusiones}

Cerro Colorado es el área funeraria de mayor extensión en la costa central peruana. Está ubicado en el extremo meridional del valle de Huaura. Entre los años 2014 y 2017, las investigaciones arqueológicas de rescate arqueológico en la Asociación de Vivienda Los Pinos (I, III, IV y V Etapa), permitieron recuperar cerca de dos mil contextos funerarios, uno de estos corresponde al fardo de un neonato conteniendo al interior un quipu sin nudos, puesto que aún no había sido utilizado.

Este personaje era un futuro quipucamayoc, quien falleció a los pocos meses de vida y fue enterrado con su futuro implemento laboral, el quipu; al parecer esta actividad era heredada de su padre. Sin embargo, este no es el primer hallazgo de quipus en este sitio, ya Julio C. Tello había estudiado otros quipus procedentes de esta área funeraria.

\section{Bibliografía}

ARELLANO, Carmen

1999 “Quipu y tocapu. Sistemas de comunicación Inca”. Los Incas. Arte y símbolos. Banco de Crédito del Perú. Lima, pp. 215-262.

ESPINOZA SORIANO, Waldemar

1997 Los Incas. Ed. Amaru. Lima, 507 pp.

MORALES CAUTI, Héctor

2009 "Informe final del Proyecto de rescate Arqueológico Complementario en la Parcela 3, sitio arqueológico de Cerro Colorado, Áreas Ocupadas II, III, IV y V, Asociaciones de Vivienda Palmeras Unidas y Cerro Colorado, distrito de Santa María, Provincia de Huaura. Informe final presentado al INC".

RADICATI DI PRIMEGLIO, Carlos

2006 Estudios sobre los quipus. Clásicos Sanmarquinos. Universidad Nacional Mayor de San Marcos. Lima, $408 \mathrm{pp}$.

RODRIGUEZ GUILLÉN, Luis

2007 "Informe final del Proyecto de Rescate Arqueológico en la Parcela 3 del sitio Cerro Colorado - Huacho”. Informe final presentado al Instituto Nacional de Cultura. 124 Pp. más planos.

ROSTWOROWSKI DE DIEZ CANSECO, María

1990 “Los kipu en la planificación Inca”. Quipu y Yupana: Colección de escritos. CONCYTEC. Lima, pp. 59-65.

TELLO, Julio C.

2015 Cuadernos de Investigación del Archivo Tello. “Arqueología del territorio Chancay”. № 11. Museo de Arqueología y Antropología, Universidad Nacional Mayor de San Marcos. Lima. 
TOSSO MORALES, Walter

2000 "Informe final del Proyecto de Estudio de Evaluación Arqueológica en la modalidad de evaluación, prospección y reconocimiento sistemático con excavaciones en el Asentamiento Humano Alberto Fujimori entregado al INC”. Informe final presentado al INC.

URTON, Gary y BREZINE, Carrie

2009 “Una tipología del khipu”. Sistemas de notación Inca: Quipu y Tocapu. Actas del Simposio Internacional. Ministerio de Cultura. Lima, pp. 43-70.

VALLEJO, Francisco

2010 "Evidencias arqueológicas de un nuevo estilo cerámico en el valle de Huaura para el Periodo Intermedio tardío: El paso del Horizonte Medio al Intermedio tardío". Arqueología en el Perú. Nuevos aportes para el estudio de las sociedades andinas. Rubén Romero y Trine Pavel editores. Lima, pp. 229 -248.

VAN DALEN LUNA, Pieter

2007 “Análisis de un quipu Tahuantinsuyo procedente de Lauri, valle de Chancay". Revista Cultural Kullpi. Investigaciones culturales en la provincia de Huaral y el Norte Chico. Año 3, № 3. Huaral, pp. $217-242$.

2015 "Julio C. Tello y las investigaciones en el territorio de la cultura Chancay". Cuadernos de Investigación del Archivo Tello. Arqueología del territorio Chancay. № 11. Museo de Arqueología y Antropología, Universidad Nacional Mayor de San Marcos. Lima, pp. 9-22.

2016 "Estrategias de dominación Tawantinsuyu en el complejo arqueologico de Lumbra, valle medio del río Chancay, provincia de Huaral". Tesis para optar el grado de Magister en Arqueología Andina. Universidad Nacional Mayor de San Marcos, Facultad de Ciencias Sociales. Lima, $455 \mathrm{pp}$.

VAN DALEN LUNA, Pieter y GRADOS RODRÍGUEZ, Hans

2014 “Los quipus del complejo arqueológico de Lumbra, Huaral”. Guara. Revista de investigación científica y cultural. Museo de Arqueología, Universidad Nacional José Faustino Sánchez Carrión. $\mathrm{N}^{\circ} 18$. Huacho, pp. $19-27$.

VAN DALEN LUNA, Pieter; CARBONEL ARANA, Dayanna

2015 "Un caso de cordoma óseo (cáncer) en individuo Chancay de Cerro Colorado, Santa María, Huaura”. Arqueología y Sociedad. № 29. Museo de Arqueología y Antropología de San Marcos. Universidad Nacional Mayor de San Marcos. Lima, pp. 167-182.

VAN DALEN LUNA, Pieter; TELLO CUADROS, Roberto; GRADOS RODRIGUEZ, Hans

2015 "Un Contexto funerario del Horizonte Medio procedente de Cerro Colorado, Huacho". Arqueología y Sociedad. № 30. Museo de Arqueología y Antropología de San Marcos. Universidad Nacional Mayor de San Marcos. Lima, pp. 407-424.

VAN DALEN LUNA, Pieter; GRADOS RODRIGUEZ, Hans; TELLO CUADROS, Roberto; VIVANCO, Iván; FLORES LIVIA, Wendy; y MARCELO GONZÁLES, Yerovi

2014 "Resultados del proyecto de rescate arqueológico en el área de la parcela 4 y 5 del sitio arqueológico Cerro Colorado, Santa María, provincia de Huaura". Kullpi. Investigaciones culturales en la provincia de Huaral y el Norte Chico. Año 7, № 7, Lima, pp. 189-216. 